\title{
Enhanced acoustic mode coupling resulting from an internal solitary wave approaching the shelfbreak in the South China Sea
}

\author{
Linus Y. S. Chiu ${ }^{\text {a) }}$ \\ Institution of Applied Marine Physics and Undersea Technology, National Sun Yat-sen University, \\ No. 70, Lienhai Road, Kaohsiung 80424, Taiwan \\ D. Benjamin Reeder \\ Department of Oceanography, Naval Postgraduate School, 833 Dyer Road, Monterey, California 93943 \\ Yuan-Ying Chang and Chi-Fang Chen \\ Department of Engineering Science and Ocean Engineering, National Taiwan University, \\ No. 1, Sec. 4, Roosevelt Road, Taipei, 10617 Taiwan \\ Ching-Sang Chiu \\ Department of Oceanography, Naval Postgraduate School, 833 Dyer Road, Monterey, California 93943 \\ James F. Lynch
Applied Ocean Physics and Engineering Department, Woods Hole Oceanographic Institution,
266 Woods Hole Road, Woods Hole, Massachusetts 02543
}

(Received 28 June 2012; revised 30 November 2012; accepted 10 January 2013)

\begin{abstract}
Internal waves and bathymetric variation create time- and space-dependent alterations in the ocean acoustic waveguide, and cause subsequent coupling of acoustic energy between propagating normal modes. In this paper, the criterion for adiabatic invariance is extended to the case of an internal solitary wave (ISW) encountering a sloping bathymetry (i.e., continental shelfbreak). Predictions based on the extended criterion for adiabatic invariance are compared to experimental observations from the Asian Seas International Acoustics Experiment. Using a mode 1 starter field, results demonstrate time-dependent coupling of mode 1 energy to higher adjacent modes, followed by abrupt coupling of mode 5-7 energy to nonadjacent modes $8-20$, produces enhanced mode coupling and higher received levels downrange of the oceanographic and bathymetric features. Numerical simulations demonstrate that increasing ISW amplitude and seafloor slope enhance the coupling of energy to adjacent and nonadjacent modes. This enhanced coupling is the direct result of the simultaneous influence of the ISW and its proximity to the shelfbreak, and, compared to the individual effect of the ISW or shelfbreak, has the capacity to scatter $2-4$ times the amount of acoustic energy from below the thermocline into the upper water column beyond the shelfbreak in realistic environments. (C) 2013 Acoustical Society of America. [http://dx.doi.org/10.1121/1.4789358]
\end{abstract}

PACS number(s): 43.30.Bp, 43.30.Zk [TFD]

Pages: 1306-1319

\section{INTRODUCTION}

Acoustic normal modes propagating in the shallow water waveguide have been shown to transfer energy between modes at frequencies of a few hundred $\mathrm{Hz}$ due to fluctuations in the structure of the shallow water waveguide, most notably by internal waves and varying bathymetry (Jensen and Kuperman, 1980; Jensen and Tindle, 1987; Chiu et al., 2004; Duda, 2004; Duda et al., 2004; Lynch et al., 2004; Duda et al., 2011). For the case of sound propagating through (perpendicular to the wavefront of) an internal solitary wave (ISW), Preisig and Duda (1997) showed that the energy shifts between modes at the steep faces of the ISW's, occurs over ranges of tens of meters, and is driven by the relative phases (differences of phases of the complex modeamplitude coefficients) of the dominant modes. The coupling of energy between modes in the shallow water waveguide

\footnotetext{
a) Author to whom correspondence should be addressed. Electronic mail: linus@mail.nsysu.edu.tw
}

results in a temporally fluctuating gain or loss of acoustic energy received at ranges of 10's of $\mathrm{km}$ from the acoustic source, depending on the source depth and the amplitude and placement of the ISW in the waveguide (Duda and Preisig, 1999).

Coupled mode theory of acoustic propagation was first developed by Pierce (1965) who employed the adiabatic separation of variables to the acoustic wave equation using range-dependent depth functions describing a locally stratified waveguide ("local modes"). The simplifying assumption of the waveguide possessing only horizontal boundaries (the "adiabatic approximation") facilitated several early applications, including work by Nagl et al. (1978) in which the adiabatic range equations are solved for general range dependence analytically in terms of Airy functions by a method using a range-segmented linearization of the environmental parameters. Rutherford and Hawker (1981) proposed a correction to conventional coupled mode theory to account for nonhorizontal boundaries. Subsequent developments in coupled mode theory include work by Evans 
(1983), Fawcett (1992), McDonald (1996), Abawi et al. (1997), Abawi (2002), and Stotts (2002).

The converse of coupled mode propagation is adiabatic mode propagation, which describes the total absence of mode coupling. It is important to predict the circumstances under which mode coupling would be expected to occur. Milder (1969) showed that mode coupling can be ignored for a sufficiently gradual range dependence and was the first to propose a criterion for the condition of adiabatic invariance of modes for particular mode pairs. However, many coastal ocean environments cannot be characterized as having gradual range dependence, prompting the need to develop the theory further for range-dependent environments. Preisig and Duda (1997) derived an adiabatic criterion for the case of an ISW in an otherwise horizontally stratified environment. The present work presents an adiabatic condition for the case of an ISW in an environment having an ISW and sloping bathymetry, and applies it to oceanographic conditions found in the South China Sea.

Very large trans-basin ISW's are common oceanographic features in the South China Sea and impact the propagation of acoustic modes (Chiu et al., 2004; Duda et al., 2004; Lynch et al., 2004). Internal tides and nonlinear internal waves are generated in the Luzon Strait during the spring and neap tides and propagate westward to the continental shelf (Ramp et al., 2004; Liu et al., 2004). The largest nonlinear internal waves, exceeding $150 \mathrm{~m}$ in amplitude, are generated during the spring tide. As the waves propagate from the deep basin to the slope and shelf, their amplitudes and horizontal scales change while trains of smaller waves are created in their wakes. Chiu et al. (2004) investigated mode coupling effects due to internal waves and bathymetric variation across the shelfbreak along one of the acoustic propagation paths in the Asian Seas International Acoustics Experiment (ASIAEX) in 2001, which had a waveguide similar to the geometry depicted in Fig. 1.

Figure 1 shows three echosounder plots of a $125 \mathrm{~m}$ amplitude transbasin ISW approaching the continental shelf of the South China Sea. The data were taken near the shelfbreak (along the $120 \mathrm{~m}$ isobath) during the Nonlinear Internal Wave Initiative Experiment (NLIWI) in 2007 (Reeder et al. 2010), and inspired this current investigation.

This paper first extends the adiabatic invariance criteria of Milder (1969) and Preisig and Duda (1997) to the case of an ISW over sloping bathymetry (Sec. II). The extended adiabatic criterion is then used to (1) investigate the adjacent and nonadjacent coupling mechanisms which contribute to enhanced mode coupling due to the combined effects of an ISW and sloping bathymetry (Sec. III), (2) examine the enhanced acoustic coupling behavior of adjacent and nonadjacent modes due to various ISW amplitudes and bathymetric slopes (Sec. IV), and (3) compare these theoretical predictions to experimental observations from the ASIAEX experiment (Sec. V). Section VI provides a summary.

\section{EXTENDED CRITERION FOR ADIABATIC INVARIANCE}

Acoustic propagation through an oceanic waveguide having a range-dependent sound speed profile can result in
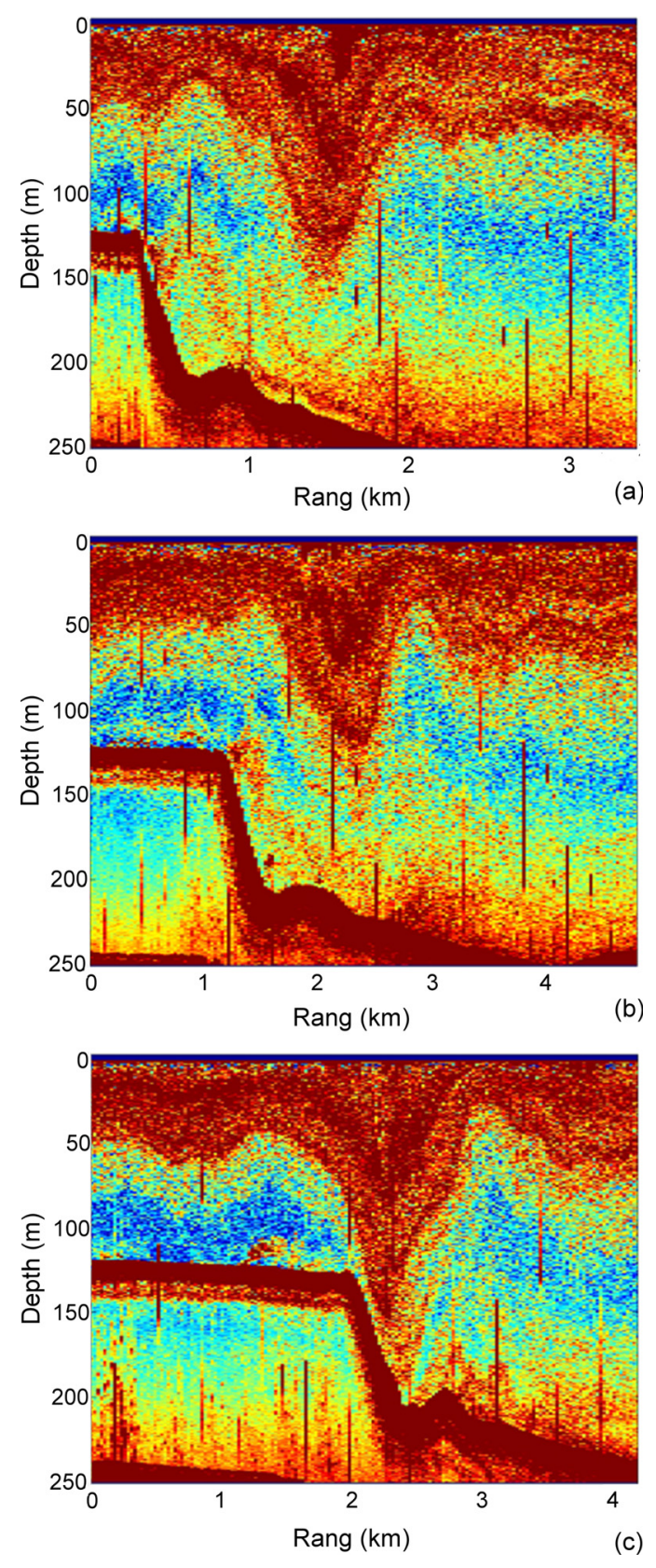

FIG. 1. (Color online) Three echo sounder plots of an ISW encountering the shelfbreak [in chronological order, (a)-(c)]. These data were collected by the Taiwanese research vessel R/V OR1 in 2007.

energy transfer between acoustic modes. Milder (1969) proposed a criterion for adiabatic invariance of modes (no mode coupling):

$$
\left|\frac{X_{i j}(x) \dot{U}_{i j}(x, z)}{\Delta E_{i j}(x)}\right| \ll \pi
$$

where

$$
\begin{aligned}
& \dot{U}_{i j}(x, z)=\int_{z} \phi_{i}(x, z) \phi_{j}(x, z) \frac{\partial U(x, z)}{\partial x} d z, \\
& \frac{\partial U(x, z)}{\partial x}=\frac{2 \omega^{2}}{\rho(z) c^{3}(x, z)} \frac{\partial c(x, z)}{\partial x},
\end{aligned}
$$


and

$$
U(x, z)=\omega^{2}\left(n_{0}^{2}-n^{2}(x, z)\right)=k_{0}^{2}-k^{2}(x, z) .
$$

Indices $i$ and $j$ indicate the $i$ th and $j$ th modes, $\phi_{i}$ and $\phi_{j}$ represent the corresponding mode functions, $c$ is the sound speed, $\omega$ is the angular frequency, $\rho$ is the density of the medium, $n$ is the slowness where $n=1 / c(x, z), k$ is the horizontal wavenumber, and $n_{0}$ and $k_{0}$ are the slowness and horizontal wavenumber at the nominal depth-averaged sound speed $\left(c_{0}\right)$ where $k_{0}=\omega n_{0}=\omega / c_{0}$. The interference length between modes $i$ and $j$ is given by $\mathrm{X}_{i j}(x) \approx 2 \pi / \Delta k_{i j}(x)$, where $\Delta k_{i j}(x)=\left|k_{i}(x)-k_{j}(x)\right|$ is the modal horizontal wavenumber difference. The energy level spacing between two modes, $\Delta E_{i j}(x)$, is stated to be $\Delta E_{i j}(x) \approx-4 \pi k_{0} /$ $X_{i j}(x) \approx-2 k_{0} \Delta k_{i j}(x)$, and is analogous to the quantity in the quantum-mechanical Schrödinger equation. $U(x, z)$ is a squared wavenumber-like term based on the Schrödinger formalism. Using the alternate expressions above for $X_{i j}(x)$, $\dot{U}(x, z)$, and $\Delta E_{i j}(x)$, Eq. (1) becomes

$$
\left|\int \phi_{i}(x, z) \phi_{j}(x, z) \frac{\partial U(x, z)}{\partial x} d z\right| \ll k_{0} \Delta k_{i j}^{2}(x) .
$$

Equation (1) states that in order for the acoustic propagation of modes to be adiabatic, the depth-weighted effective change in $U(x, z)$ occurring over a mode interference length (or a classical ray period) must be small relative to the intermodal energy level spacing. As the depth-integrated horizontal sound speed gradient increases over a ray period $\left[X_{i j}(x)\right]$, the adiabatic invariance will be violated and coupling of energy from mode $i$ to mode $j$ will be more likely. Equation (5) indicates that the likelihood of mode coupling from mode $i$ to mode $j$ increases with decreasing wavenumber difference or when the depth-weighted projection of mode $i$ to mode $j$ is large. Milder (1969) also emphasizes the point that the ability of the horizontal gradient in $U(x, z)$ to couple the modes depends entirely on its vertical structure:

$$
\left(\frac{\partial n^{2}}{\partial x}\right)_{i j}=\int \phi_{i} \frac{\partial n^{2}}{\partial x} \phi_{j} d z
$$

No coupling occurs in gradients that are independent of depth because of mode orthogonality.

The present oceanographic feature of interest that provides the spatial distribution of sound speed indicated in Eq. (1) is the ISW, which has been represented by the Korteweg-de Vries (KdV) equation in previous studies (Liu, 1988; Preisig and Duda, 1997; Colosi et al., 2001; Shroyer et al., 2011). Preisig and Duda (1997) expressed the shallow water ISW displacement of the boundary in the hyperbolic secant form

$$
\eta(x)=-a \operatorname{sech}^{2}\left[\left(x-R_{i}\right) / L\right],
$$

where $a$ and $L$ are the amplitude and horizontal scale of the ISW, respectively, and $R_{i}$ indicates the location of the wave trough propagating in the $x$-direction. The expression in Eq. (7) was applied to Eq. (1), and the adiabatic criterion for a three-layered, flat bottom waveguide with ISW's was derived as (Preisig and Duda, 1997)

$$
a \cos \theta(x) \frac{4 \omega c_{0} \Delta c}{\Delta z} \frac{U_{i j}(a, x / L) \operatorname{sech}^{2}(x / L) \tanh (x / L)}{\Delta k_{i j}^{2}(x / L)} \ll L,
$$

where $\Delta c$ and $\Delta z$ are the sound speed and depth differences between the upper and lower layers in the water column and $\theta(x) \in[-\pi / 2, \pi / 2]$ is the angle between the ISW and the acoustic wave vectors. The $\cos \theta$ term is consistent with the expectation that mode coupling is most likely when the ISW and acoustic horizontal wave vectors are colinear but is not likely at large $\theta(x)$ when horizontal refraction is expected to be the dominant propagation mechanism. Equation (8) states that for a given acoustic angular frequency $(\omega)$ and ISW amplitude $(a)$ and length $(L)$, the likelihood of mode coupling increases with decreasing wavenumber difference or increasing strength of water column stratification (i.e., sound speed gradient of the transition (middle) layer).

In this paper, the adiabatic criterion is extended to an ISW in a waveguide with linearly sloping bathymetry. Consider the three-layer water column shown in Fig. 2, consisting of an isospeed $\left(c_{1}\right)$ upper layer; a constant gradient middle (transition) layer having constant thickness $\left(z_{u^{-}} z_{l}\right)$ and bounded by sound speeds $c_{1}$ above and $c_{2}$ below, perturbed by $\eta(x)$; and a lower layer which includes the lower water column and seabed. The lower water column layer possesses a constant linear sound speed gradient bounded by sound speeds $c_{2}$ above and $c_{3}(z)$ at the water-sediment interface. The seabed is modeled as a fluid sediment half-space of constant sound speed $\left(c_{4}\right)$ and density $\left(\rho_{4}\right)$, and contains a section of inclined bathymetry described by a sloping function, $\zeta(x)$ :

$$
\begin{aligned}
\zeta(x)= & 0\left\{H[x]-H\left[x-R_{S}\right]\right\}+b\left(x-R_{S}\right)\left\{H\left[x-R_{s}\right]\right. \\
& \left.-H\left[x-R_{e}\right]\right\}+\Lambda H\left[x-R_{e}\right],
\end{aligned}
$$

where $R_{S}$ is the starting point of the inclination on the $\mathrm{x}$-axis with slope $b$, and $R_{e}$ is the ending point of the inclination after which the thickness of the water column is $z_{l}-z_{b}-\Lambda$. The first and second layers of this environment are identical to those of Preisig and Duda (1997).

The sound speed profile is parameterized as

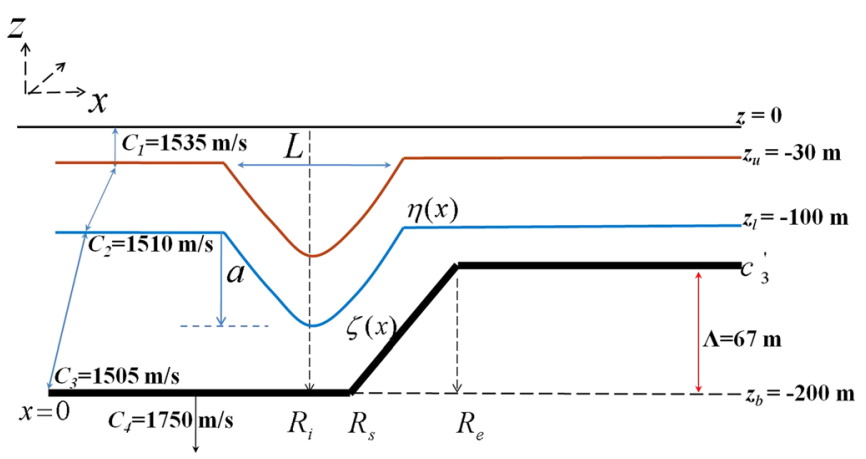

FIG. 2. (Color online) Environmental model used for analysis. The environmental parameters include water depths $z$, sound speeds $c$, ISW amplitude $a$ and width $L$, thermocline displacement $\eta(x)$, bottom slope $\zeta(x)$, altitude of shelf $\Lambda$, and distance along the horizontal $(R)$. The parameter values shown in the figure are used in numerical modeling and also listed in Table I. 


$$
\begin{array}{rlrl}
c(x, z)= & c_{1}\left\{H[-z]-H\left[\left(z_{u}+\eta(x)\right)-z\right]\right\} & \text { (the 1st layer) } \\
& +\left\{\frac{c_{2}-c_{1}}{z_{\ell}-z_{u}}\left[z-\left(z_{u}+\eta(x)\right)\right]+c_{1}\right\} & \text { (the 2nd layer) } \\
& \times\left\{H\left[\left(z_{u}+\eta(x)\right)-z\right]-H\left[\left(z_{l}+\eta(x)\right)-z\right]\right\} & \text { (the 3rd layer) } \\
& +\tilde{c}(x, z)\left\{H\left[\left(z_{l}+\eta(x)\right)-z\right]-H\left[\left(z_{b}+\varsigma(x)\right)-z\right]\right\} \\
& +c_{4} H\left[\left(z_{b}+\varsigma(x)\right)-z\right],
\end{array}
$$

where $H[z]$ is the Heaviside step function and $\tilde{c}(x, z)$ is given below. In the first layer, the gradient of the sound speed is given by

$$
\frac{\partial c(x, z)}{\partial x}=\underline{-c_{1} \frac{d \eta(x)}{d x} \delta\left[\left(z_{u}+\eta(x)\right)-z\right]},
$$

where $\delta$ is the Dirac delta function. In the second layer, the gradient of sound speed is given by

$$
\begin{aligned}
\frac{\partial c(x, z)}{\partial x}= & -\frac{c_{2}-c_{1}}{z_{\ell}-z_{u}} \frac{d \eta(x)}{d x}\left\{H\left[\left(z_{u}+\eta(x)\right)-z\right]-H\left[\left(z_{l}+\eta(x)\right)-z\right]\right\} \\
& +\left\{\frac{c_{2}-c_{1}}{z_{\ell}-z_{u}}\left[z-\left(z_{u}+\eta(x)\right)\right]+c_{1}\right\} \frac{d \eta(x)}{d x} \delta\left[\left(z_{u}+\eta(x)\right)-z\right] \\
& -\left\{\frac{c_{2}-c_{1}}{z_{\ell}-z_{u}}\left[z-\left(z_{u}+\eta(x)\right)\right]+c_{1}\right\} \frac{d \eta(x)}{d x} \delta\left[\left(z_{l}+\eta(x)\right)-z\right] \\
&
\end{aligned}
$$

The third layer includes the sediment of constant sound speed $\left(c_{4}\right)$ and the lower portion of the water column which follows the distribution

$$
\tilde{c}(x, z)=c_{2}+\frac{c_{3}-c_{2}}{z_{b}-\left(z_{l}+\eta(x)\right)}\left[z-\left(z_{l}+\eta(x)\right)\right] .
$$

The background, or unperturbed $[\eta(x)=0]$, sound speed profile in this lower portion of the water column is a linear function between $c_{2}$ and $c_{3}$. The gradient of the sound speed in this third layer is typically nonzero and is given as

$$
\begin{aligned}
\frac{\partial c(x, z)}{\partial x}= & \frac{\partial \tilde{c}(x, z)}{\partial x}\left\{H\left[\left(z_{l}+\eta(x)\right)-z\right]-H\left[\left(z_{b}+\varsigma(x)\right)-z\right]\right\} \\
& \frac{+\tilde{c}(x, z) \frac{d \eta(x)}{d x} \delta\left[\left(z_{l}+\eta(x)\right)-z\right]}{} \\
& -\tilde{c}(x, z) \frac{d \varsigma(x)}{d x} \delta\left[\left(z_{b}+\varsigma(x)\right)-z\right]+c_{4} \frac{d \varsigma(x)}{d x} \delta\left[\left(z_{b}+\varsigma(x)\right)-z\right] .
\end{aligned}
$$

The terms $\delta\left[\left(z_{u}+\eta(x)\right)-z\right]$ and $\delta\left[\left(z_{l}+\eta(x)\right)-z\right]$ are nonzero only at the interfaces $z=z_{u}+\eta(x)$ and $z=z_{l}+\eta(x)$; the horizontal sound speed gradients occurring at both interfaces are cancelled by combining the single-underscored terms in Eqs. (11) and (12) for interface $z=z_{u}+\eta(x)$ and the double-underscored terms Eqs. (12) and (14) for interface $z=z_{l}+\eta(x)$.

Thus, the gradient of the sound speed in the first layer is zero and the gradient in the second layer [Eq. (12)] is reduced to

$$
\frac{\partial c(x, z)}{\partial x}=-\frac{c_{2}-c_{1}}{z_{\ell}-z_{u}} \frac{d \eta(x)}{d x} \times\left\{H\left[\left(z_{u}+\eta(x)\right)-z\right]-H\left[\left(z_{l}+\eta(x)\right)-z\right]\right\} .
$$




$$
\frac{\partial \tilde{c}(x, z)}{\partial x}=\frac{-\left(c_{3}-c_{2}\right) \frac{d \eta(x)}{d x}\left[z_{b}-\left(z_{l}+\eta(x)\right)\right]+\left(c_{3}-c_{2}\right)\left[z-\left(z_{l}+\eta(x)\right)\right] \frac{d \eta(x)}{d x}}{\left[z_{b}-\left(z_{l}+\eta(x)\right)\right]^{2}},
$$

Eq. (14) reduces to

$$
\begin{aligned}
\frac{\partial c(x, z)}{\partial x}= & \frac{\left(c_{3}-c_{2}\right)\left(z-z_{b}\right)}{\left[z_{b}-\left(z_{l}+\eta(x)\right)\right]^{2}} \frac{d \eta(x)}{d x}\left\{H\left[\left(z_{l}+\eta(x)\right)-z\right]-H\left[\left(z_{b}+\varsigma(x)\right)-z\right]\right\} \\
& +\left\{c_{4}-\tilde{c}(x, z)\right\} \frac{d \varsigma(x)}{d x} \delta\left[\left(z_{b}+\varsigma(x)\right)-z\right] .
\end{aligned}
$$

Expanding Eq. (5) and setting the limits of integration for the second and third layers:

$$
\left|\int_{z_{l}+\eta(x)}^{z_{u}+\eta(x)} \phi_{i}(x, z) \phi_{j}(x, z) \frac{\partial U(x, z)}{\partial x} d z+\int_{z_{b}+\varsigma(x)}^{z_{l}+\eta(x)} \phi_{i}(x, z) \phi_{j}(x, z) \frac{\partial U(x, z)}{\partial x} d z\right| \ll k_{0} \Delta k_{i j}^{2}(x) .
$$

Using Eqs. (3) and (15) for the second layer in the first term of Eq. (18) gives

$$
\int_{z_{l}+\eta(x)}^{z_{u}+\eta(x)} \phi_{i}(x, z) \phi_{j}(x, z) \frac{\partial U(x, z)}{\partial x} d z=\frac{-2 \omega^{2}\left(c_{2}-c_{1}\right)}{\left(z_{l}-z_{u}\right)} \frac{\partial \eta(x)}{\partial x} \int_{z_{l}+\eta(x)}^{z_{u}+\eta(x)} \frac{\phi_{i}(x, z) \phi_{j}(x, z)}{\rho_{w}(z) c^{3}(x, z)} d z
$$

and using Eqs. (3) and (17) for the third layer in the second term in Eq. (18) gives

$$
\begin{aligned}
\int_{z_{b}+\varsigma(x)}^{z_{l}+\eta(x)} \phi_{i}(x, z) \phi_{j}(x, z) \frac{\partial U(x, z)}{\partial x} d z= & 2 \omega^{2}\left(c_{3}-c_{2}\right) \frac{d \eta(x)}{d x} \int_{z_{b}+\varsigma(x)}^{z_{l}+\eta(x)} \frac{\phi_{i}(x, z) \phi_{j}(x, z)\left(z-z_{b}\right)}{\rho_{w}(z) c^{3}(x, z)\left[z_{b}-\left(z_{l}+\eta(x)\right)\right]^{2}} d z \\
& +\left.\frac{2 \omega^{2} \phi_{i}(x, z) \phi_{j}(x, z)\left[c_{4}-\tilde{c}(x, z)\right]}{\rho_{B}(z) c^{3}(x, z)} \frac{d \varsigma(x)}{d x}\right|_{z=z_{b}+\varsigma(x)}
\end{aligned}
$$

where $\rho_{w}(z)$ is the water column density and $\rho_{B}(z)$ is the sediment density starting at the water-seabed interface. Finally, combining Eqs. (18)-(20) yields the extended adiabatic criterion for a waveguide having both an ISW and sloping bathymetry:

$$
\frac{2 \omega^{2}\left|\psi_{i j}(x, z)\right|}{k_{0} \Delta k_{i j}^{2}(x)} \ll 1,
$$

where $\rho_{w}(z)$ is assumed to be $1.0 \mathrm{~g} / \mathrm{cm}^{3}$ and

$$
\begin{aligned}
\psi_{i j}(x, z)= & \frac{d \eta(x)}{d x} W_{i j}(x, z) \\
& +\left.\frac{d \varsigma(x)}{d x} \frac{\left(c_{4}-\tilde{c}(x)\right) \phi_{i}(x, z) \phi_{j}(x, z)}{\rho_{B}(z) c^{3}(x, z)}\right|_{z=z_{b}+\varsigma(x)}
\end{aligned}
$$

and

$$
\begin{aligned}
W_{i j}= & \frac{c_{1}-c_{2}}{z_{u}-z_{l}} \int_{z_{l}+\eta(x)}^{z_{u}+\eta(x)} \frac{\phi_{i}(x, z) \phi_{j}(x, z)}{c^{3}(x, z)} d z \\
& -\frac{c_{2}-c_{3}}{\left[z_{b}-\left(z_{l}+\eta(x)\right)\right]^{2}} \\
& \times \int_{z_{b}+\varsigma(x)}^{z_{l}+\eta(x)} \frac{\left(z-z_{b}\right) \phi_{i}(x, z) \phi_{j}(x, z)}{c^{3}(x, z)} d z .
\end{aligned}
$$

Using the ISW shape and slope functions given in Eqs. (7) and (9) yields

$$
\begin{aligned}
& \frac{\partial \eta(x)}{\partial x}=-\frac{2 a}{L} \operatorname{sech}^{2}\left(\frac{x-R_{i}}{L}\right) \tanh \left(\frac{x-R_{i}}{L}\right), \\
& \frac{\partial \zeta(x)}{\partial x}=b\left\{H\left[x-R_{s}\right]-H\left[x-R_{e}\right]\right\} .
\end{aligned}
$$

Rewriting Eq. (21) in terms of the ISW amplitude $a$, horizontal scale $L$ and position $R_{i}$, the bathymetric slope $b$, and the position of the slope $\left[R_{s}, R_{e}\right]$ gives 

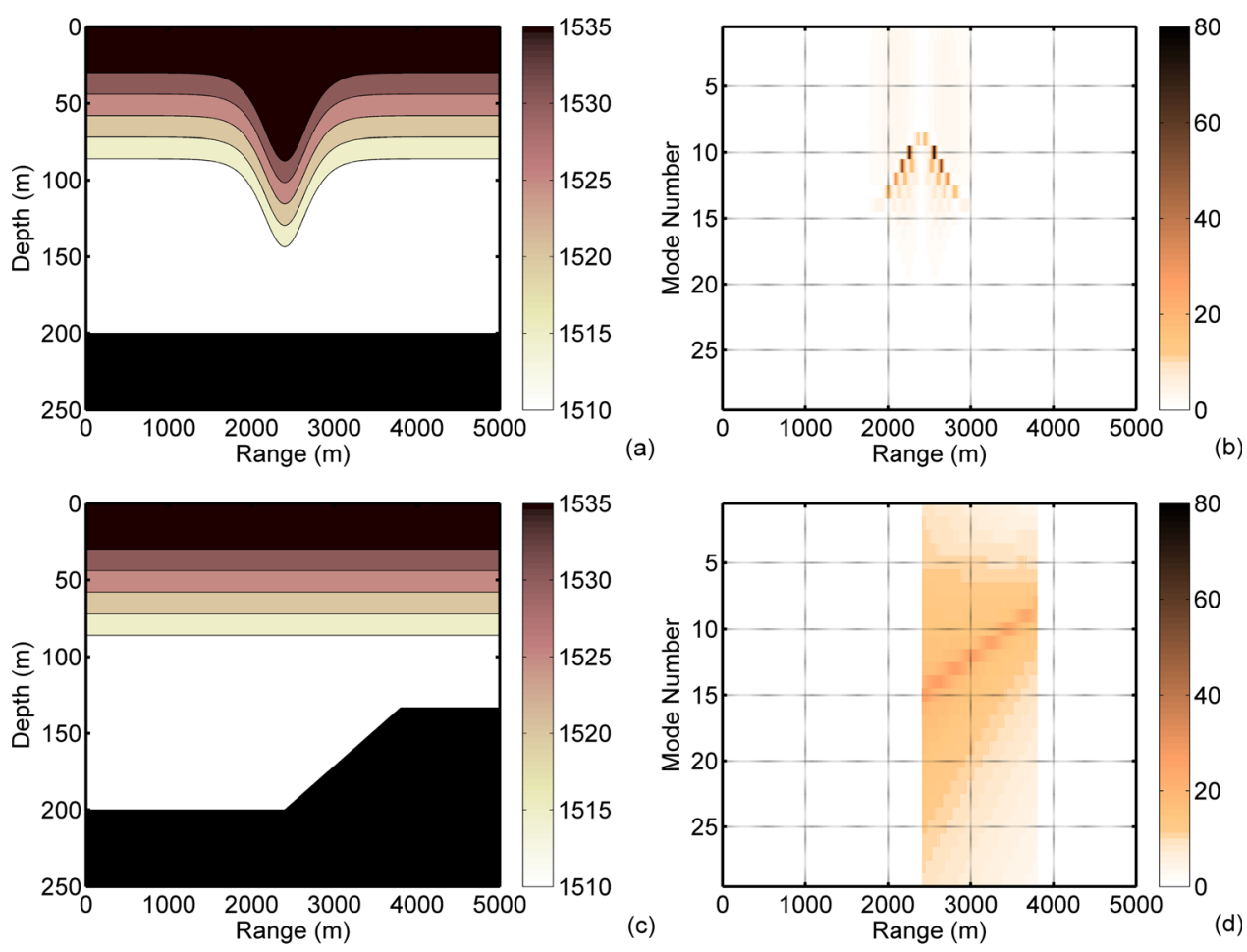

FIG. 3. (Color online) Adjacent mode coupling strength for three environments. Case ISW: flat seabed with an ISW [(a) and (b)]; case SB: sloping seabed with no ISW [(c) and (d)]; and case ISW-SB: sloping seabed with an ISW [(e) and (f)]. Panels (a), (c), and (e) are sound speed profiles and bottom bathymetry; panels (b), (d), and (f) show the calculated adjacent mode coupling strength $\left[\beta_{i j}(x)\right]$ for each case as a (c)
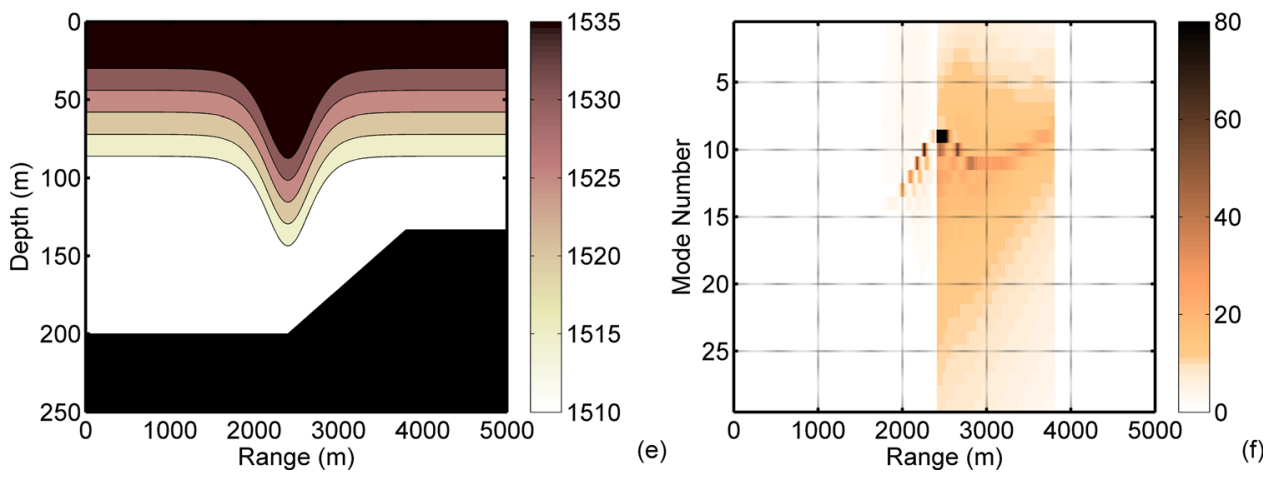
function of mode number and range.

$$
\frac{2 \omega^{2}\left|\frac{2 a}{L} \operatorname{sech}^{2}\left(\frac{x-R_{i}}{L}\right) \tanh \left(\frac{x-R_{i}}{L}\right) W_{i j}(x, z)+b V_{i j}(x, z)\right|}{k_{0} \Delta k_{i j}^{2}(x)} \ll 1,
$$

where

$$
\begin{aligned}
V_{i j}(x, z)= & \left\{H\left[x-R_{s}\right]-H\left[x-R_{e}\right]\right\} \\
& \times\left.\frac{\left(c_{4}-\tilde{c}(x, z)\right)\left(\phi_{i}(x, z) \phi_{j}(x, z)\right)}{\rho_{B}(z) c^{3}(x, z)}\right|_{z=z_{b}+\varsigma(x)} .
\end{aligned}
$$

Following the notation of Preisig and Duda (1997), the left hand side of Eq. (25) can be designated the coupling strength, $\beta_{i j}(x)$, which is the new, extended adiabatic criterion for a waveguide modulated by range-dependent variations in both bathymetry and the water column sound speed. The coupling strength of $\beta_{i j}(x)$ is determined by both the modal wavenumber difference $\left[\Delta k_{i j}(x)\right]$ in the denominator and the depthintegrated horizontal gradient of the sound speed in the numerator. Equation (25) is physically consistent with Eq. (1); mode coupling is shown to be more likely if $\Delta k_{i j}(x)$ is small or if the horizontal gradient of the sound speed is large.
In the following section, the modal coupling strength $\beta_{i j}(x)$ is examined via numerical experimentation to gain insight into the dominant physical mechanisms of mode coupling and the environmental conditions under which mode coupling is expected to occur.

\section{ENHANCED COUPLING}

The extended adiabatic criterion derived in Sec. II is applied here to study the enhanced coupling effect of an environment containing ISW's approaching a shelfbreak as shown in Fig. 1. In this section, three numerical cases are presented to explore the individual effects of an ISW or shelfbreak, as well as the effect of both together: (1) a flat seabed with an ISW ["case ISW," Fig. 3(a)], (2) a shelfbreak with no ISW ["case SB," Fig. 3(c)], and (3) a shelfbreak with an ISW [“case ISW-SB," Fig. 3(e)].

\section{A. Implementation}

In all cases, the background sound speed profile (SSP) is set as a downward-refracting profile, and the fluctuations induced by the ISW's are treated as a perturbation of the thermocline. The environment consists of a three-layer, 
horizontally stratified water column $\left(c_{1}=1535 \mathrm{~m} / \mathrm{s}\right.$, $c_{2}=1510 \mathrm{~m} / \mathrm{s}, \quad c_{3}=1505 \mathrm{~m} / \mathrm{s}, \rho_{w}=1.0 \mathrm{~g} / \mathrm{cm}^{3}$ ) as shown in Fig. 2. The top layer has a thickness of $30 \mathrm{~m}$ with a constant sound speed of $c_{1}$, the second layer spans water depths of 30$100 \mathrm{~m}$ with a constant sound speed gradient, and the third layer occupies the remainder of the water column from $100 \mathrm{~m}$ to the seabed which varies from 200 to $133 \mathrm{~m}$. The seabed is modeled as a penetrable half-space, composed of loose sand (bottom sound speed $c_{b}=1750 \mathrm{~m} / \mathrm{s}$, bottom attenuation $\alpha_{b}=0.5 \mathrm{~dB} / \lambda$, bottom density $\rho_{b}=1.8 \mathrm{~g} / \mathrm{cm}^{3}$ ). The sloping portion has an angle with the horizontal of approximately $\sim 2.73^{\circ}(1 / 21)$ and extends from $x=2.4$ to $3.8 \mathrm{~km}$.

For case ISW and case ISW-SB, an ISW is superimposed on the background SSP. The shallow-water ISW displacement of the boundary is described by Eq. (7), with parameters $a$ and $L$ initially given as 57.5 and $350 \mathrm{~m}$, respectively. The setting of $a$ and $L$ makes the ISW in the modeled waveguide extend vertically $\sim 157 \mathrm{~m}$ from the sea surface and horizontally a wavelength of $\sim 1 \mathrm{~km}$, which is similar to what is seen in Fig. 1. In case ISW-SB, the ISW position is defined as the location of the wave trough, which coincides with the beginning of the shelfbreak $\left(x=R_{i}=R_{s}=2.4 \mathrm{~km}\right)$. The modeled geometry and acoustic propagation conditions, as described here and specified in Table I, are consistent with environmental conditions observed during several acoustic field experiments conducted in the South China Sea during the past decade.

The acoustic propagation simulations are computed at $400 \mathrm{~Hz}$ with a mode 1 starter field which represents lowmode-dominated propagation conditions in a downward refracting environment with a deep acoustic source. In addition to computing the mode functions and $\beta_{i j}(x)$ for each numerical case, transmission loss and mode fields are also computed. Applying "A Modal Spectrum of the PE Field (MOSPEF)" (Shang and Wang, 1993), the solutions of sound field $[P(r, z)]$ are computed by a 2D wide-angle PE code and are projected on mode amplitudes as

$$
A_{n}(r)=\int P(r, z) \phi_{n}(r, z) d z,
$$

TABLE I. Environmental parameters.

\begin{tabular}{lc}
\hline \hline Slope & $\theta=2.73^{\circ}(1 / 21)$ \\
Range to starting point of slope & $R_{s}=2.4 \mathrm{~km}$ \\
Height of shelf relative to $z_{b}$ & $\Lambda=67 \mathrm{~m}$ \\
Amplitude of ISW & $a=57.5 \mathrm{~m}$ \\
Horizontal scale of ISW & $L=350 \mathrm{~m}$ \\
Location of the ISW trough & $R_{i}=2.4 \mathrm{~km}$ \\
Depth of sound speed interface & $z_{u}=-30 \mathrm{~m}$ \\
& $z_{l}=-100 \mathrm{~m}$ \\
Sound speed in water column & $z_{b}=-200 \mathrm{~m}$ \\
& $c_{1}=1535 \mathrm{~m} / \mathrm{s}$ \\
& $c_{2}=1510 \mathrm{~m} / \mathrm{s}$ \\
Water density & $c_{3}=1505 \mathrm{~m} / \mathrm{s}$ \\
Seabed sound speed & $\rho_{\mathrm{w}}=1.0 \mathrm{~g} / \mathrm{cm}^{3}$ \\
Seabed attenuation & $c_{4}=1750 \mathrm{~m} / \mathrm{s}$ \\
Seabed density & $\alpha_{b}=0.5 \mathrm{~dB} / \lambda$ \\
\hline \hline
\end{tabular}

where $\phi_{n}(z, r)$ is the local mode function generated by FEMODE (Collins, 1988).

\section{B. Adjacent mode coupling}

Figure 3 shows the modeled environment (left column) and adjacent mode $(i, j=i+1)$ coupling strength (right column) for each of the three cases. A relatively large value of $\beta_{i j}(x)$ indicates that if there is energy in mode $i$, there is greater relative likelihood of energy transfer to mode $j=i+1$. The behavior of $\beta_{i j}(x)$ in these environments allows convenient categorization of modes into three groups: low modes (modes 1-8), medium modes (9-14), and high modes (15 and higher).

A number of observations of adjacent mode coupling behavior in these environments can be made based on Fig. 3 . For all three cases, adiabatic mode propagation is expected before the ISW and after the shelfbreak $\left[\beta_{i j}(x)<1\right]$, with greater relative likelihood of coupled mode propagation in the vicinity of the ISW and shelfbreak $\left[\beta_{i j}(x)>1\right]$. Strong mode coupling is evident in all three cases in the vicinity of the ISW and shelfbreak for the medium modes, with values of $\beta_{i j}(x)$ greater than 50. For case ISW [panels (a) and (b)], modes 1-8 have values of $\beta_{i j}(x)$ between 1 and 20 and modes 9-14 have values greater than 20, while the higher modes have smaller values, indicating mode-selective coupling-coupling between higher adjacent modes is less likely than in the low and medium modes. For case SB [panels (c) and (d)] and case ISW-SB [panels (e) and (f)], adjacent mode coupling is expected for all mode pairs due to decreasing water depth. In case SB, the largest values of $\beta_{i j}(x)$ appear as a monotonically increasing striation for the medium modes. While the symmetry in the shape of the ISW in case ISW [Fig. 3(b)] creates symmetry in $\beta_{i j}(x)$ consistent with the results of Preisig and Duda (1997), the symmetry no longer holds for case ISW-SB. The values and symmetric pattern of $\beta_{i j}(x)$ found in case ISW are modified by the presence of the shelfbreak in case ISW-SB [cf. Figs. 3(b) and 3(f)]; specifically, (1) the values in the first half of the ISW are identical in case ISW and case ISW-SB; (2) case ISW-SB values of $\beta_{i j}(x)$ exceed those of case ISW and case SB between $x=2400$ and 3000; (3) a transition zone of elevated $\beta_{i j}(x)$ values appears between the second halves of the ISW and shelfbreak, centered on $x=3000 \mathrm{~m}$, indicating increased likelihood of coupling between modes 11 and 12 that did not exist in cases ISW or SB individually; and (4) the enhanced values of $\beta_{i j}(x)$ in the second half of the ISW are followed by the monotonically increasing striation for the medium modes associated with the top half of the shelfbreak as seen in case SB.

Recalling Eq. (25) — the value of $\beta_{i j}(x)$ is determined by the modal wavenumber difference $\left[\Delta k_{i j}(x)\right]$ in the denominator and the depth-integrated horizontal gradient of the sound speed in the numerator. The numerator is mainly composed of two terms - the first term containing $W_{i j}$ represents the effect of the ISW and the second term containing $V_{i j}$ represents the effect of the shelfbreak. In case ISW, the second term containing $V_{i j}$ is zero. Figure 4 illustrates the behavior of the wavenumber difference (top panels) and values of $\beta_{i j}(x)$ (bottom 

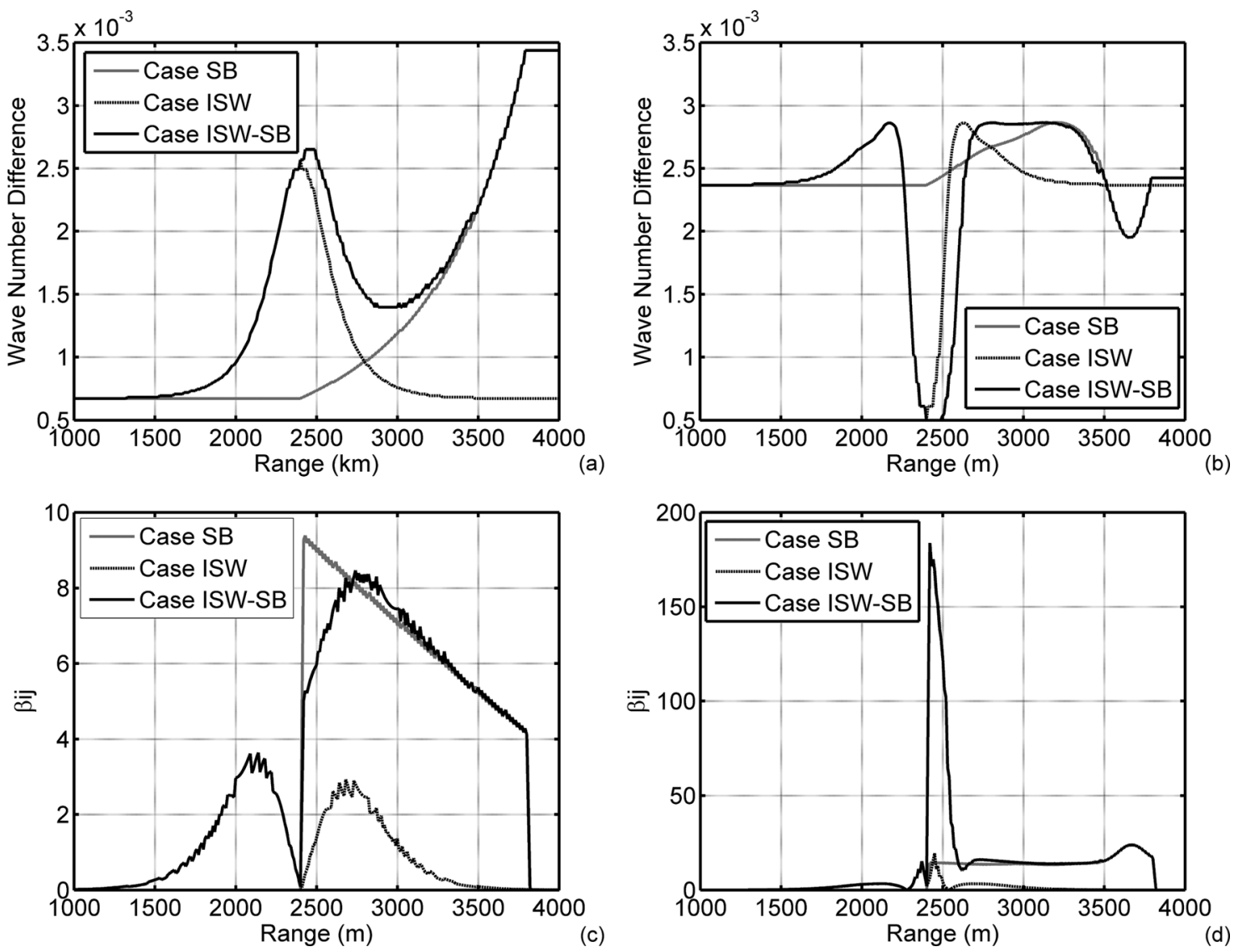

FIG. 4. Wavenumber difference (top) and mode coupling strength $\beta_{i j}(x)$ (bottom) as a function of range for the three environments for exemplar adjacent mode pairs 1-2 [(a) and (c)] and 9-10 [(b) and (d)].

panels) as a function of range for the three environments for exemplar adjacent mode pairs 1-2 [(a) and (c)] and 9-10 [(b) and (d)]. Comparison of panels (a) and (b) prompt several observations: (1) For case ISW in panel (a), the value of $\Delta k_{i j}(x)$ is initially less than 1 and has zero slope, then increases with a strong gradient to the peak corresponding to the trough of the ISW at $x=2400 \mathrm{~m}$, then decreases with a strong negative gradient to the same minimum value after the ISW. The increased values of $\beta_{i j}(x)$, as seen in panel (c), occur between the smallest and largest values of $\Delta k_{i j}(x)$ when the sound speed gradient is greatest on the steep faces of the ISW, consistent with the findings of Preisig and Duda (1997). Even though $\Delta k_{i j}(x)$ in the denominator of Eq. (25) is increasing, the numerator containing the effect of the sound speed gradient is the dominant contributor to elevated values of $\beta_{i j}(x)$ which result in mode coupling. (2) For cases ISW and ISW-SB in panel (b), the decrease in $\Delta k_{i j}(x)$ contributes to the increase in $\beta_{i j}(x)$ for mode pair 9-10, but it is also evident that the elevated values of $\beta_{i j}(x)$ occur [panel (d)] when the horizontal gradient of the sound speed is greatest due to the simultaneous influence of the second half of the ISW and the first half of the shelfbreak. (3) The wavenumber difference in case ISW-SB for both mode pairs [1-2 and 9-10, panels (a) and (b)] is comparable to that of case ISW up to $x=2900 \mathrm{~m}$, indicating that $\Delta k_{i j}(x)$ is the minor contributor to the enhanced mode coupling seen between $x=2400$ and $2900 \mathrm{~m}$ in Fig. 3 .

The above three observations demonstrate that the sound speed gradient as represented in the numerator of
Eq. (25) is the dominant contributor to the enhancement of the coupling strength. Furthermore, as the acoustic energy first encounters the change in slope at the shelfbreak, it is the $\partial \zeta(x) / \partial x$ term in Eqs. (22) and (24) that produces a discontinuous increase in $\beta_{i j}(x)$ and continued elevated values of $\beta_{i j}(x)$ in cases SB and ISW-SB between $x=2400$ and $3800 \mathrm{~m}$ compared to that of case ISW which is driven largely by the $\partial \eta(x) / \partial x$ term, Eqs. (22) and (24).

Figure 4 illustrates the individual and combined effects of the ISW and shelfbreak. The dominant mode coupling mechanism for adjacent mode pair $1-2$ is the shelfbreak as evidenced by the comparable values of $\beta_{i j}(x)$ in cases SB and ISW-SB for $x>2400 \mathrm{~m}$. However, the values of $\beta_{i j}(x)$ in case ISW-SB for mode pair 9-10 between $x=2400$ and $2600 \mathrm{~m}$ appear to be anomalously large compared to cases ISW and SB, which suggests that there may be an additional mechanism at work that explains the very strong coupling occurring in the medium modes in case ISW-SB [Fig. 3(f)].

\section{Nonadjacent mode coupling}

Figure 5 shows modal content as a function of range for the first 30 modes propagating from a mode 1 starter field for case ISW (a), case SB (b), and case ISW-SB (c). Panels (a) and (b) demonstrate that as the acoustic energy encounters the ISW or shelfbreak, the lower mode energy is coupled to higher adjacent modes, as was seen in 

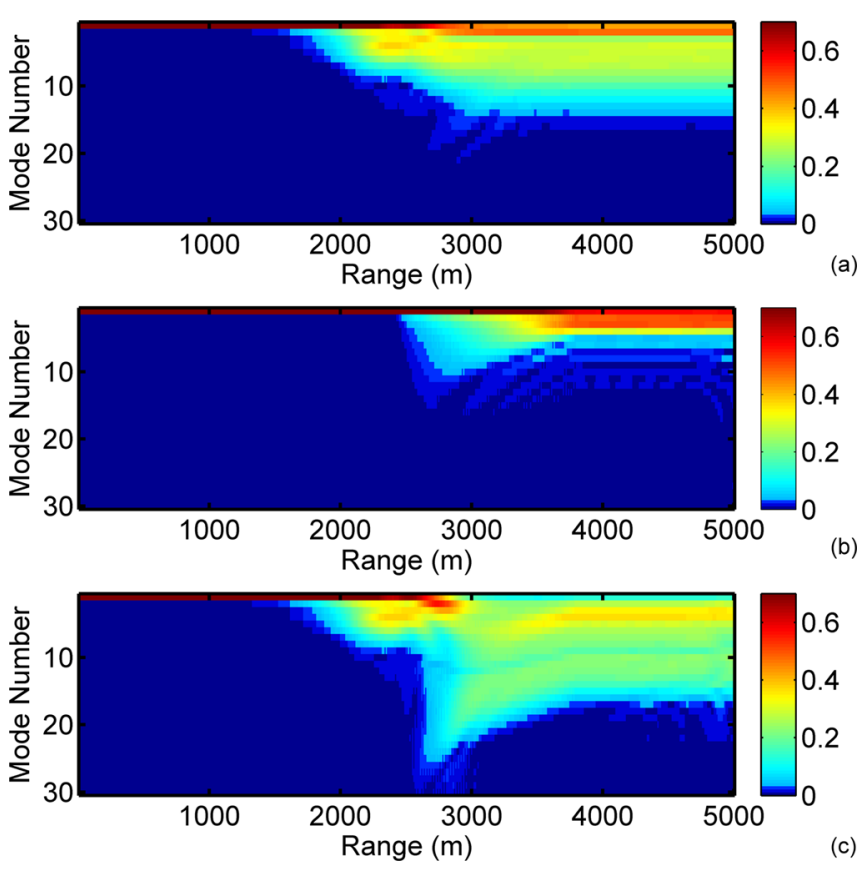

FIG. 5. (Color online) Modal content (normalized amplitude) as a function of range for the first 30 modes propagating from a mode 1 starter field for case ISW (a), case SB (b), and case ISW-SB (c).

Figs. 3(b) and 3(d). For case ISW, continuous coupling occurs from mode 1 to adjacent modes 2-7 within the first half of internal wave, and continued coupling up to mode 11 while most of the energy couples back down to modes 1 and 2 after the ISW. For case SB, energy scatters from mode 1 to modes 2-7 up to the mid-point of the shelfbreak, then resides in modes $1-5$ after the shelfbreak. Little to no energy is coupled to medium (8-15) and higher $(>15)$ modes in either case ISW or case SB. In case ISW-SB, the mode 1 energy couples to modes 2-7 in the first half of the ISW (as was observed in case ISW); then energy appears abruptly and briefly in modes 8-25 when it encounters the ISW trough at the beginning of the shelfbreak; finally, the energy settles into modes 15 and below after the shelfbreak. The energy that propagates beyond the shelfbreak consists of modes 2 and above, with most of the energy residing in modes 3 and 4; very little of the mode 1 energy that existed before the ISW and shelfbreak remains after the shelfbreak. The coupling of higher modes to lower and medium modes between $x=3000-3500$ is consistent with the high values of $\beta_{i j}(x)$ at $x=3000-3500$ in Fig. 3(f). Physically, these higher modes are analogous to higher angle rays resulting from enhanced downward refraction caused by the thermocline depression in the first half of the ISW; these higher angle rays then reflect to even higher angles on the shelfbreak. After the shelfbreak, the energy no longer experiences the depressed thermocline or sloping bathymetry and consists of lower and medium modes as a result of attenuation and coupling.

The most significant feature in Fig. 5(c) is the abrupt coupling of energy from lower modes to nonadjacent medium and higher modes as the energy encounters the second half of the ISW over the sloping bathymetry between $x=2400$ and $3000 \mathrm{~m}$. Close inspection of panel (c) reveals strong coupling from mode 1 to adjacent modes 2 and 3 in the second half of the ISW in addition to the aforementioned coupling to modes $2-7$, followed by the abrupt coupling to nonadjacent medium and higher modes. The energy that abruptly couples to the nonadjacent medium and higher modes apparently comes from the energy in modes 2-7 that was first coupled from mode 1 in the first half of the ISW. Results (not shown here) verified that values of $\beta_{i j}(x)$ for nonadjacent mode pairs $1-15$ to $1-20$ are nearly zero for case ISW-SB, confirming that the energy that abruptly appears in the medium and higher modes does not come from mode 1. In order for this enhanced coupling to occur, the ISW must be approaching the shelfbreak such that the energy coupled from mode 1 to modes $2-7$ by the first half of the ISW is available for coupling to medium and higher modes by the simultaneous influence of the second half of the ISW and first half of the shelfbreak. If the ISW is not in the vicinity of the shelfbreak, most of the energy in modes 2-7 couples back down to modes 1 and 2 as is seen for case ISW in panel (a). It appears that this nonadjacent mode coupling resulting from the joint effect of the ISW approaching the shelfbreak is the physical mechanism responsible for the anomalously strong medium mode coupling behavior first illustrated Fig. 3(f).

To further elucidate the coupling to nonadjacent modes, Fig. 6 presents the magnitude of $\beta_{i j}(x)$ for exemplar nonadjacent mode pairs 7-10, 10-14, and 14-18 for $x=1000$ $4000 \mathrm{~m}$ for case ISW [panel (a)], case SB [panel (b)], and case ISW-SB [panel (c)]. For $x<2400 \mathrm{~m}$, the values $\beta_{i j}(x)$ are very small for all three environments. For case ISW, maximum values of $\beta_{i j}(x)$ occur at the leading and trailing edges of the ISW but remain less than unity throughout, indicating that nonadjacent mode coupling for all three mode pairs is not likely, consistent with Fig. 5(a). For case SB, $\beta_{i j}(x)$ immediately jumps to values of $1-1.5$ at the beginning of the shelfbreak, generally decreases to the end of the slope, and then returns to zero after the shelfbreak. Close inspection of Fig. 5(b) reveals some small amount of energy in modes 7 and 10, but none in mode 14 and above. For case ISW-SB, values of $\beta_{i j}(x)$ are identical to those in case ISW up to $x=2400 \mathrm{~m}$ and are identical to those of case SB after $x=3300 \mathrm{~m}$. Between $x=2400$ and $3300 \mathrm{~m}$, while values of $\beta_{i j}(x)$ for nonadjacent mode pair 14-18 are slightly lower than for case SB and exhibit the same pattern, $\beta_{i j}(x)$ for nonadjacent mode pairs 7-10 and 10-14 exhibit sinusoidal patterns and are elevated in value above those for cases ISW and SB. The first peak of 2.2 in the sinusoidal pattern coincides with trailing edge of the ISW [cf. panel (a)]; the second peak of 3.0 occurs at $x=2700 \mathrm{~m}$ and coincides with the abrupt coupling of energy from lower modes to medium and higher modes as seen in Fig. 5(c).

There are two key elements to this enhanced mode coupling mechanism: (1) the coupling from mode 1 to modes 2-7 in the first half of the ISW provides the energy required to support nonadjacent mode coupling from modes 2-7 to modes 8-25; and (2) the second half of the ISW must be positioned above the first half of the shelfbreak in order for this nonadjacent mode coupling to occur and propagate enhanced high modal energy onto the shelf. 

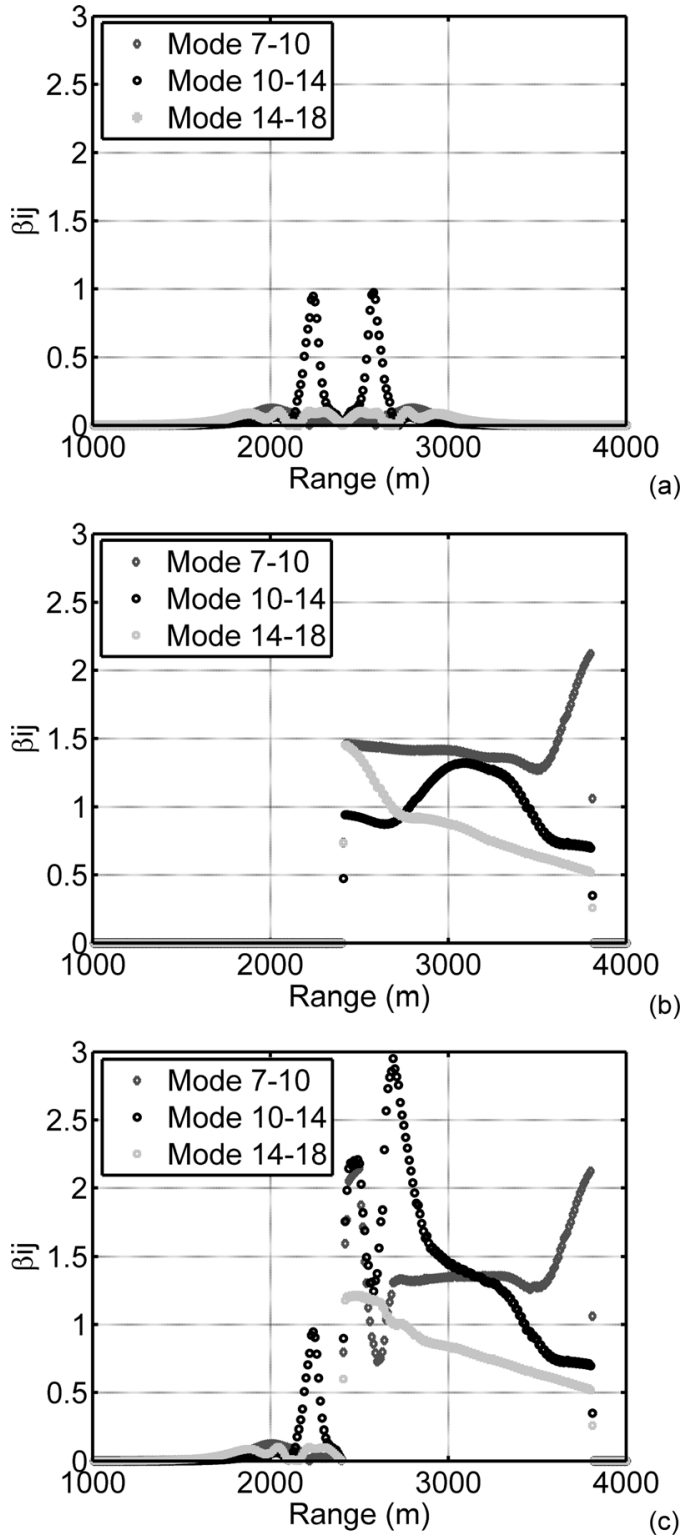

FIG. 6. Mode coupling strength $\beta_{i j}(x)$ as a function of range for exemplar nonadjacent mode pairs 7-10, 10-14, and 14-18 for case ISW (a), case SB (b), and case ISW-SB (c).

\section{ENERGY ENHANCEMENT DUE TO MODE COUPLING AT THE SHELFBREAK}

In this modeling effort, the starter field consists of mode 1 only. Even in the case of adiabatic mode propagation, it is expected that this mode 1 energy will experience some redistribution in the water column due to the effective narrowing of the waveguide by the shelfbreak; therefore, it is important to discriminate between energy variation due to adiabatic mode propagation alone and energy variation due to enhanced mode coupling. These differences are examined by calculating the transmission loss (TL) for case ISW-SB using adiabatic mode theory (Jensen et al., 1994) and coupled mode theory, which are shown in Fig. 7. In the case of adiabatic propagation [panel (a)], while the depth-dependent distribution of mode 1 energy changes with the narrowing of channel, mode 1 energy remains confined to depths below $70 \mathrm{~m}$ before and after the SB in this downward refracting
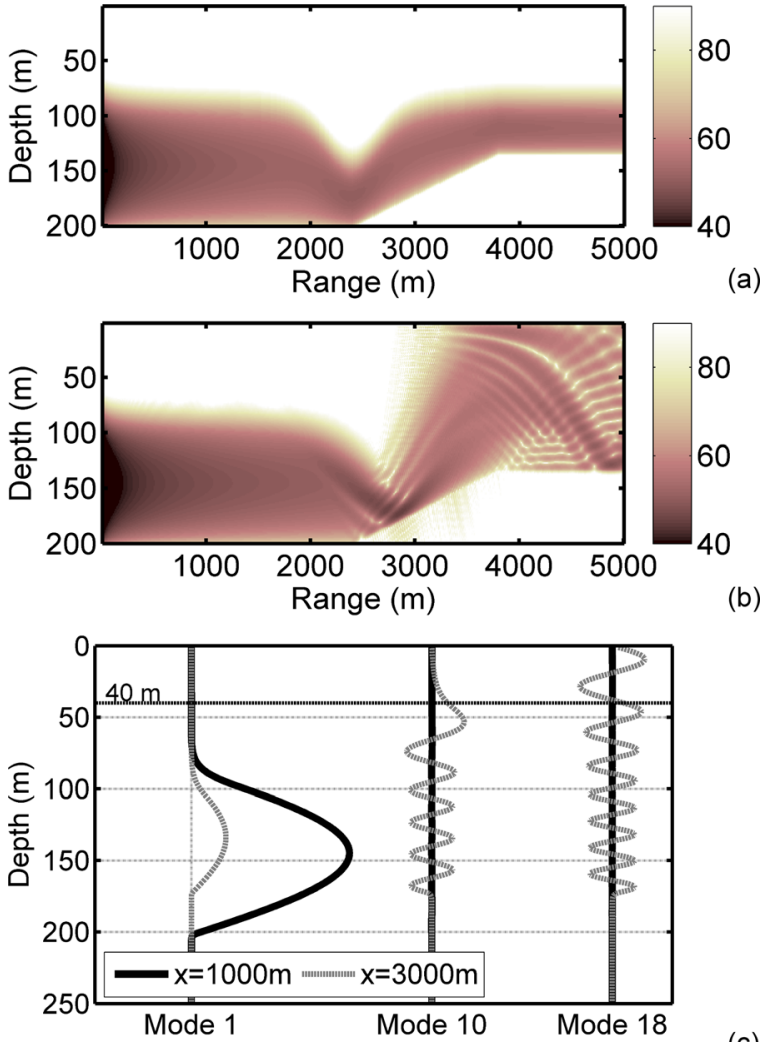

(c)

FIG. 7. (Color online) Modeled TL for case ISW-SB for (a) adiabatic propagation and (b) coupled-mode propagation, both with a mode 1 starting field. Mode shapes (all normalized by the same factor) as a function of depth for modes 1,10 , and 18 for case ISW-SB. Solid lines represent the mode functions at $x=1000 \mathrm{~m}$ (before ISW and SB), and dashed lines represent the mode functions at $x=3000 \mathrm{~m}$.

environment. On the contrary, when accounting for mode coupling [panel (b)], energy is coupled from mode 1 to higher modes after interaction with the ISW and SB. Figure 7(c) shows three selected modes (all normalized by the same factor) as a function of depth at two ranges for case ISW-SB under coupled-mode propagation conditions: before the ISW and SB ( $x=1000 \mathrm{~m}$, solid lines), and on the SB ( $x=3000 \mathrm{~m}$, dashed lines). Modes 1,10 , and 18 represent three dominant modes contributing to sound intensity in the lower, middle and upper water column, respectively. As seen in panel (a), mode 1 energy experiences some vertical redistribution and loss, but is constrained to the lower water column before and after the ISW and SB. As demonstrated in the previous section, mode 1 energy encountering the ISW and SB couples continuously up to mode 11 , followed by nonadjacent mode coupling to higher modes; this redistribution of energy from the lower water column to the middle and upper water column is evident in modes 10 and 18 at $x=3000 \mathrm{~m}$, consistent with panel (b) on and downrange of the shelfbreak.

Figure 8 compares energy variation due to adiabatic mode propagation alone and energy variation due to enhanced mode coupling due to ISW's of various amplitudes (a) with fixed horizontal scale $(L)$ and various bathymetric slopes $(b)$ in terms of depth-averaged acoustic energy anomaly (EA) in $\mathrm{dB}$ vs range $(x)$ for the top portion $(0-40 \mathrm{~m})$ of the waveguide. EA is the difference between sound intensity level for the modeled environment and the "background" 

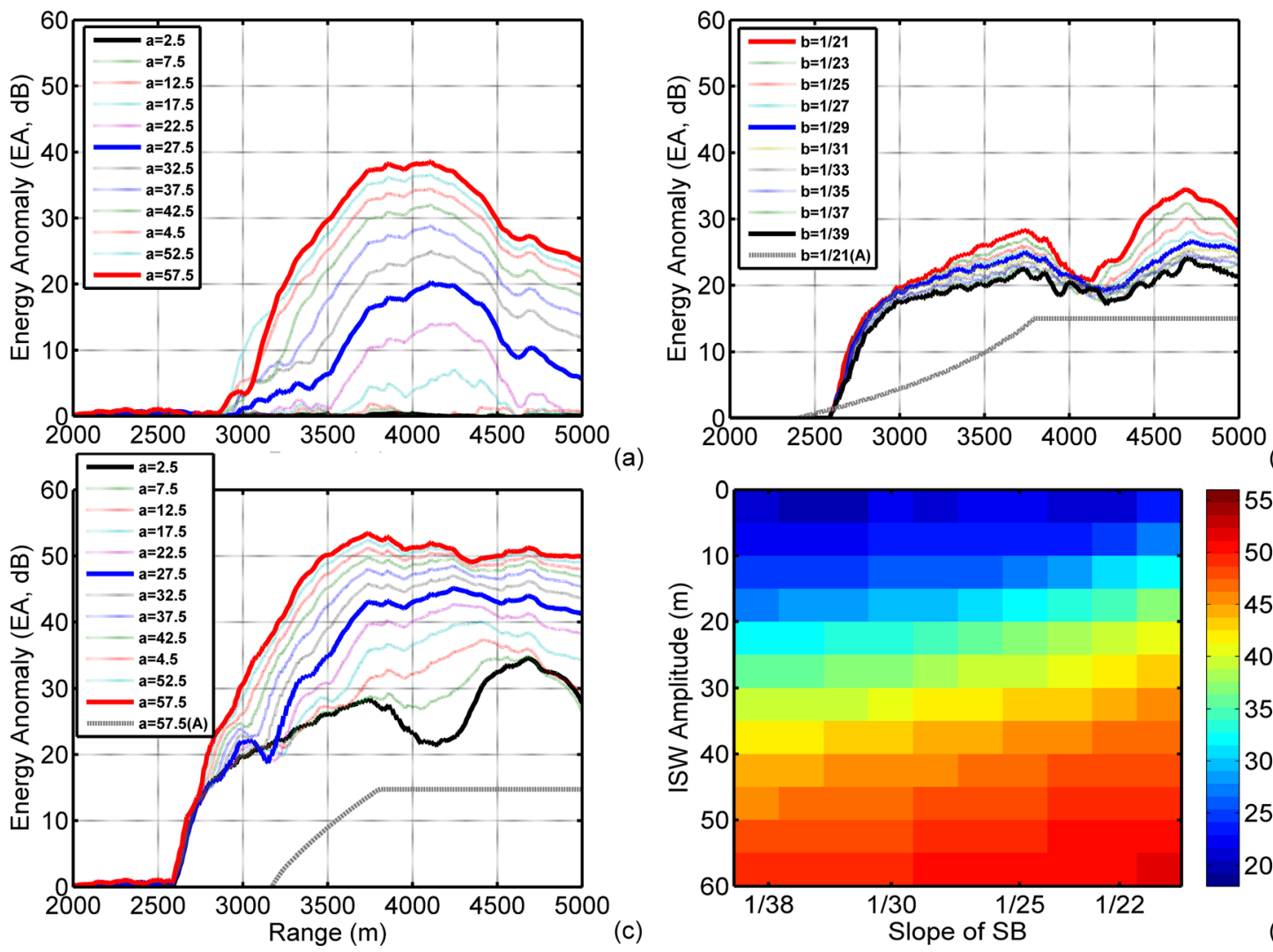

(a)

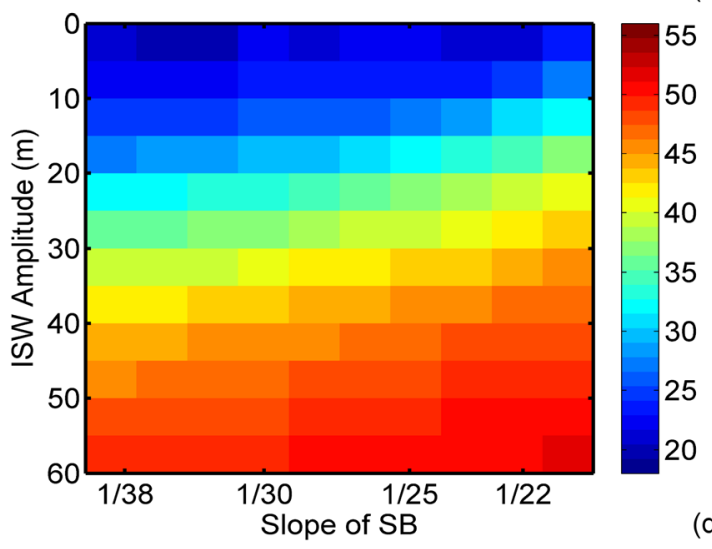

(b)

FIG. 8. (Color online) Depth-averaged acoustic energy anomaly (EA) in dB for the top portion ( $0-40 \mathrm{~m}$ ) of the waveguide for various ISW amplitudes and
bathymetric slopes. EA is the difference between sound intensity level for the computed environment and the "background" sound intensity level (a waveguide with no ISW and no shelfbreak). Panel (a) shows EA above the thermocline vs range (m) for ISW amplitudes $a=2.5-57.5 \mathrm{~m}$ in $5 \mathrm{~m}$ increments (case ISW); panel (b) shows EA above the thermocline vs range for bathymetric slopes $b=1 / 39$ to $1 / 21$ (case SB); panel (c) shows EA above the thermocline vs range for ISW amplitudes, $a=2.5-7.5 \mathrm{~m}$ in $5 \mathrm{~m}$ increments with fixed slope $b=1 / 21$ (case ISW-SB); and panel (d) shows EA (dB) above the thermocline at $x=4000 \mathrm{~m}$ as a function of ISW amplitude and slope. The thick dashed lines in panels (b) and (c) show EA above the thermocline vs range for adiabatic propagation for case SB with slope $b=1 / 21$ and for case ISW-SB with ISW amplitude $a=57.5 \mathrm{~m}$ and slope $b=1 / 21$, respectively.

sound intensity level (a waveguide with no ISW and no shelfbreak). The acoustic intensity is integrated over the top $40 \mathrm{~m}$ of the waveguide to represent the average amount of energy that is scattered by the ISW and/or shelfbreak from the deep mode- 1 source near the seabed to the surface layer above the thermocline.

Figure 8(a) shows EA above the thermocline vs range (m) for ISW amplitudes $a=2.5-57.5 \mathrm{~m}$ in $5 \mathrm{~m}$ increments (case ISW). Energy does not appear above the thermocline until $x \sim 2900$, approximately $500 \mathrm{~m}$ after the ISW trough. EA at the largest modeled ISW amplitude of $57.5 \mathrm{~m}$ reaches a maximum of $\sim 38 \mathrm{~dB}$ at $x=3800-4200 \mathrm{~m}$, approximately $2 \mathrm{~km}$ after the energy encounters the first half of the ISW. EA in the upper $40 \mathrm{~m}$ of the water column is low ahead of the ISW because the mode 1 energy is trapped below the thermocline. Once the ISW is encountered, energy is initially downwardrefracted into steeper angles in the first half of the ISW, then subsequently scattered into higher modes by higher-angle interaction with the seabed and the second half of the ISW, resulting in the increase in observable energy above the thermocline near the end of the second half of the ISW. Beyond the peak at $x \sim 4000$, the medium and higher mode energy in the upper portion of the water column decreases due largely to modal interference and, to a lesser degree, attenuation.

Figure 8 (b) presents EA above the thermocline vs range (m) for slopes $b=1 / 39-1 / 21$ (case SB). Energy does not appear above the thermocline until $x \sim 2600$, approximately $200 \mathrm{~m}$ after the beginning of the shelfbreak. EA, containing a strong modal interference pattern, reaches a maximum of $\sim 34 \mathrm{~dB}$ at $x \sim 4700 \mathrm{~m}$ (at the largest modeled slope of 1/21), approximately $2.3 \mathrm{~km}$ after the energy encounters the beginning of the shelfbreak and $\sim 900 \mathrm{~m}$ after the end of the shelfbreak. Most of the energy that appears in the upper water column is scattered there while traversing the shelfbreak. The largest modeled slope of $1 / 21$ increases EA by only about $10 \mathrm{~dB}$ above that produced by the smallest slope of $1 / 39$. Also shown in panel (b) is the adiabatic variation in EA (thick dashed line) expected from the compression of the mode 1 energy due to the narrowing of the waveguide for $b=1 / 21$. Comparison of EA for the coupled mode propagation vs adiabatic propagation (thick dashed line) demonstrates a maximum difference of $\sim 20 \mathrm{~dB}$ on the SB at $x \sim 4700 \mathrm{~m}$.

Figure 8(c) illustrates EA above the thermocline vs range (m) for ISW amplitudes $a=2.5-57.5 \mathrm{~m}$ and slope $b=1 / 21$ (case ISW-SB). EA at the largest modeled ISW amplitude of $57.5 \mathrm{~m}$ reaches a maximum of $\sim 54 \mathrm{~dB}$ at $x \sim 3700 \mathrm{~m}$, near the end of the shelfbreak. Beyond the peak at $x \sim 3700$, the energy in the upper portion of the water column maintains a relatively stable level of $\sim 50 \mathrm{~dB}$ to $x>5000 \mathrm{~m}$, due to the combined contributions of the ISW and shelfbreak. Similar to panel (b), EA for adiabatic 
propagation for case ISW-SB with ISW amplitude $a=57.5 \mathrm{~m}$ and slope $b=1 / 21$ is shown. Comparison of EA for the coupled mode propagation vs adiabatic propagation (thick dashed line) shows a sustained difference of $\sim 35 \mathrm{~dB}$ on the shelfbreak, demonstrating that mode coupling is the dominant physical mechanism contributing to the energy in the upper $40 \mathrm{~m}$ of the water column.

Figure 8(d) illustrates EA above the thermocline at $x=4000 \mathrm{~m}$ as a function of ISW amplitude $(a=2.5-57.5 \mathrm{~m})$ and bathymetric slope $(b=1 / 39-1 / 21)$. Figure 8 demonstrates, as expected, that the presence of an ISW or significant change in seabed slope (e.g., shelfbreak) causes energy to be transferred to higher modes and significantly increases the amount of energy in the upper water column that otherwise would be trapped below the thermocline. The simultaneous influence of the ISW and shelfbreak (case ISW-SB) has the capacity, as modeled here in these idealized cases under coupled-mode conditions, to contribute an additional $\sim 10-15 \mathrm{~dB}$ above case ISW and $\sim 15-20 \mathrm{~dB}$ above case SB to the depth-averaged energy in the upper portion of the water column just beyond the shelfbreak.

Depicted in Fig. 9 is the EA in the upper portion of the water column $(0-40 \mathrm{~m})$ at a point downrange from the shelfbreak for case ISW-SB as a function of ISW position relative to the source position. In this case, the modeled environment, possessing an ISW of $57.5 \mathrm{~m}$ amplitude and a shelfbreak slope of $1 / 21$, has been expanded to $14.5 \mathrm{~km}$ between the source (at $x=0 \mathrm{~m}$ ) and receiver with the shelfbreak spanning $x=7500-8900 \mathrm{~m}$. As the center of the ISW moves from $x=1500 \mathrm{~m}$ to $x=8000 \mathrm{~m}$ in $500 \mathrm{~m}$ increments, the EA above the thermocline at $x=14.5 \mathrm{~km}$ exhibits a fluctuating pattern resulting from ISW-induced mode coupling and modal interference. Before the ISW reaches the shelfbreak, EA averages $45 \mathrm{~dB}$; once the trough of the ISW reaches a point $500 \mathrm{~m}$ before the beginning of the shelfbreak, EA increases to $52 \mathrm{~dB}$, approximately $5 \mathrm{~dB}$ above the previous peaks and approximately $7 \mathrm{~dB}$ above the average value of EA. This demonstrates the second key element of the enhanced mode coupling mechanism discussed in the previous section-it is not until the ISW is in close proximity to

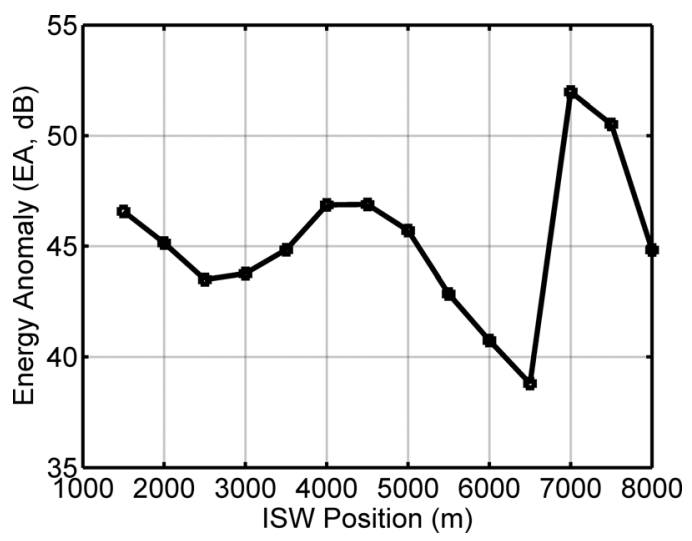

FIG. 9. Depth-averaged acoustic energy anomaly (EA) in $\mathrm{dB}$ at $x=14500 \mathrm{~m}$ for the top portion $(0-40 \mathrm{~m})$ of the waveguide as a function of ISW position (moving toward shelf) with a fixed ISW amplitude of $57.5 \mathrm{~m}$ and a fixed slope of $1 / 21$. The sound source is at $x=0$ and the shelfbreak spans $x=7500-8900 \mathrm{~m}$. the shelfbreak that the transfer of energy between nonadjacent modes occurs, resulting in a doubling or quadrupling of energy above the thermocline downrange from the oceanographic and bathymetric features.

\section{VERIFICATION WITH IN SITU EXPERIMENTAL DATA}

One of the components of the ASIAEX experiment in 2001 was conducted in the South China Sea to investigate acoustic propagation on the continental shelf and in the vicinity of the shelfbreak (Lynch et al., 2004). Analysis of these ASIAEX data provides in situ experimental evidence of the enhanced mode coupling effect.

Panels (a) and (b) of Fig. 10 illustrate the experimental geometry. A $31.3 \mathrm{~km}$ across-shelf acoustic transect was defined by the $400 \mathrm{~Hz}$ source moored near the bottom at a water depth of $337 \mathrm{~m}$ and the receiving vertical line array (VLA) moored at a depth of $124 \mathrm{~m}$. The source transmitted 88 pseudo-random noise ( $\mathrm{m}$-sequence) signals at $400 \mathrm{~Hz}$ for $7.5 \mathrm{~min}$ at $30 \mathrm{~min}$ intervals. The 16 -element VLA had a vertical aperture from 42 to $121 \mathrm{~m}$ water depth. The shelfbreak was located approximately $26 \mathrm{~km}$ from the source (Newhall et al., 2001).

The temperature data were collected at two-minute intervals at the environmental moorings, Env-350, Env-200, and Env-120, at depths of 350, 200, and $120 \mathrm{~m}$, respectively. The environmental moorings possessed temperature and pressure sensors spanning most of water column. Ten temperature sensors were also deployed on the VLA (Newhall et al., 2001). Figure 10(c) presents the temperature data recorded by the three environmental moorings on May 8th, from 0700 to 1500. A large amplitude ISW entered the acoustic propagation path at $\sim 0800$, passed Env-200 near the shelfbreak at 1230, and exited the acoustic path at $\sim 1500$. The internal waves evolve as they propagate over the continental slope and onto the shelf, due to water column mixing and boundary interaction (Yang et al., 2004 and Ramp et al., 2004). The large ISW in the top panel is seen to develop into a train of nonlinear internal waves in the middle panel; by the time the wave energy reaches Env-120 well onto the shelf, the wave energy has been attenuated and scattered into multiple high-frequency waves. Figure 10(b) shows the data-based environmental model (Chiu et al., 2004; Reeder et al., 2010) used in the numerical simulation in this section, based on the temperature record from Env200. About $8 \mathrm{~h}$ of temperature data are used to construct the ISW environmental model consisting of sound speed profiles computed from a standard, empirically derived equation (Mackenzie, 1981).

Figure 10(d) presents the sound intensity level (SIL) recorded by the top hydrophone on the VLA at $42 \mathrm{~m}$ water depth (dots). A peak in the SIL is clearly observed at 1245 , coinciding with the time that the ISW reached the shelfbreak. It is expected that this enhancement in SIL is the result of the simultaneous influence of the ISW and sloping bathymetry. To verify this expectation, the SIL at the top hydrophone on the VLA is computed for the period centered on this event with environmental input depicted in Fig. 10(b). The observed SIL exhibits a $5 \mathrm{~dB}$ peak above the mean SIL and a $2-3 \mathrm{~dB}$ 


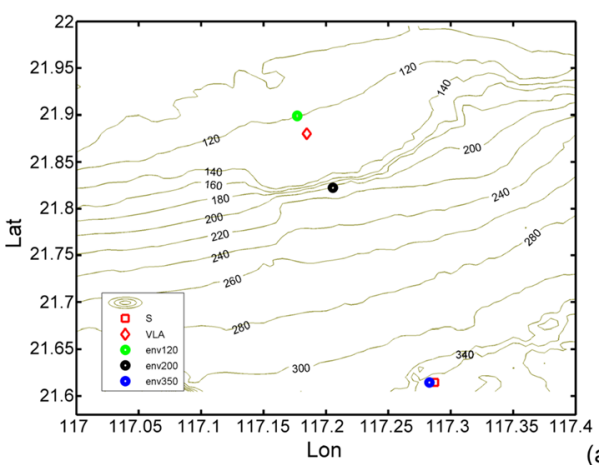

(a)
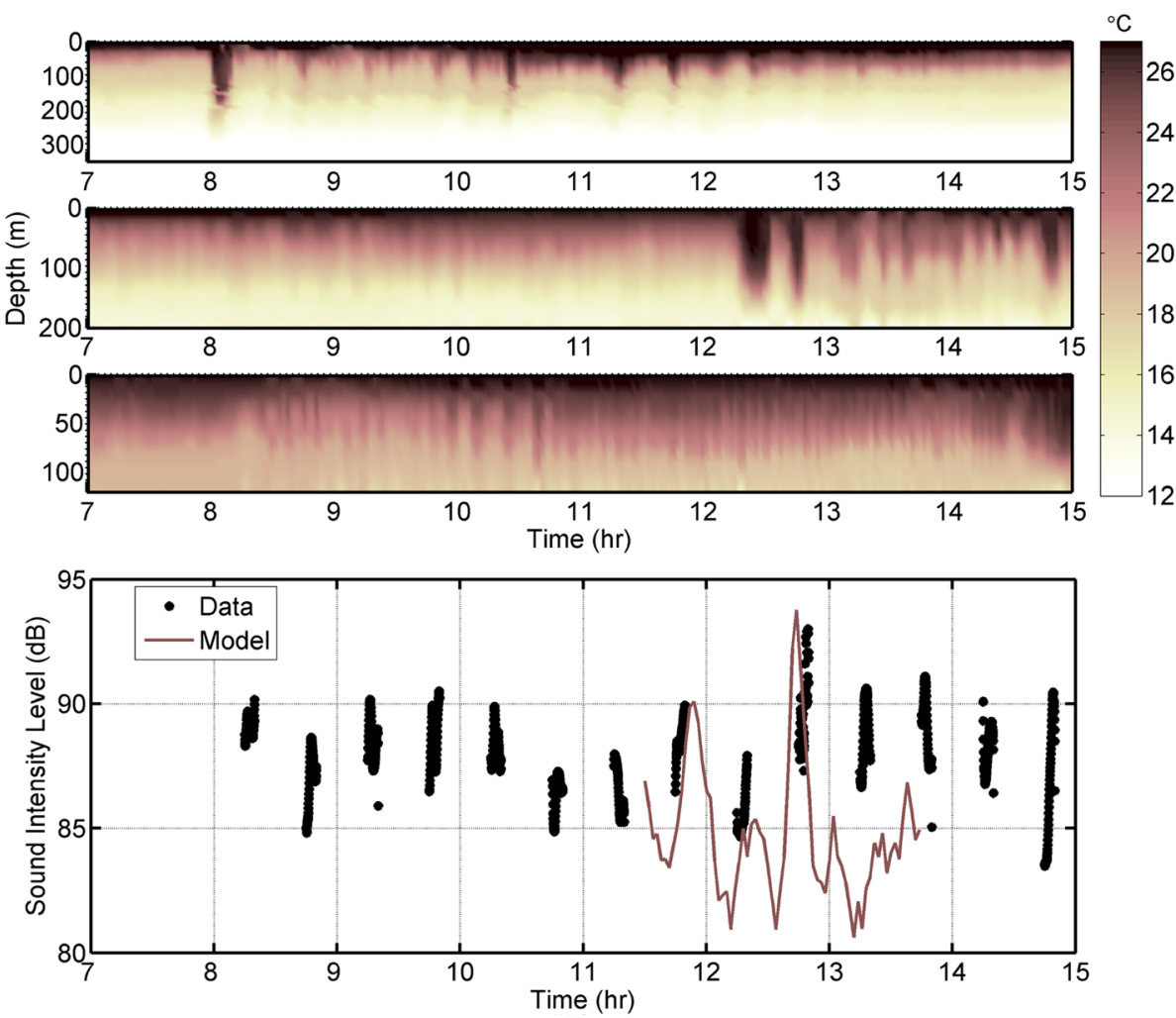

FIG. 10. (Color online) Data from the ASIAEX field experiment in 2001 in the South China Sea: (a) geographic locations of moored instrumentation; (b) acoustic track viewed from north to south on May 8; (c) temperature data collected between the VLA and the southerly source from 0700 to 1500 on environmental moorings Env350 (top panel), Env200 (middle panel), and Env120 (bottom panel); (d) observed SIL (dB) at the top hydrophone of the VLA at $42 \mathrm{~m}$ water depth (dots) and the computed SIL (dB) for the same hydrophone (solid line) using the modeled environment [panel (b)] based on data from Env-200 [panel (c)]. peak above the other peaks in the time series; the modeled SIL (solid line) exhibits similar structure, value and time of occurrence of the enhanced reception as the experimental data, with a $6 \mathrm{~dB}$ peak above the mean SIL and a $3 \mathrm{~dB}$ peak above the other large peak near 1200 in the computed time series. There is excellent model-data agreement prior to 1300 as the ISW approaches the shelfbreak. The agreement deteriorates after the ISW passes the shelfbreak due to the fact that the ISW evolves into smaller-amplitude, high-frequency internal waves on the continental shelf [cf. Fig. 10(c)], which is not adequately represented in the modeled environment. The model-data agreement prior to 1300 [panel (d)], corroborated by its similarity and consistency with Fig. 9, provides a strong indication that the enhanced reception at 1245 is due to the joint mode coupling mechanism presented in this paper.

\section{CONCLUSION}

The enhanced acoustic coupling effect due to the simultaneous influence of an ISW and sloping bathymetry of a shelfbreak is examined by (1) broadening the theory of
Preisig and Duda (1997) to create an extended adiabatic criterion for mode coupling for the case of an ISW above a bathymetric slope [Eq. (25)], (2) numerical simulations to examine the physical coupling mechanisms responsible for the enhanced mode coupling, and (3) verification by comparison model predictions to in situ experimental data. The ISW and shelfbreak decrease the wavenumber difference between modes [the denominator of Eq. (25)] and increase the depthintegrated effective change in sound speed [the numerator of Eq. (25)]. The combination of both effects produces enhanced values of $\beta_{i j}(x)$ in the second half of the ISW, and enhanced values of $\beta_{i j}(x)$ in the zone between the second halves of the ISW and shelfbreak.

In this paper, the starter field of mode 1 is emphasized to replicate the conditions during the ASIAEX experiment in the South China Sea (Chiu et al., 2004) in which most of the energy propagating from the near-bottom acoustic source energy was observed to be trapped in mode 1; and to investigate the combined effect of the ISW and shelfbreak on mode coupling in this observed environment. The mode 1 acoustic energy originally constrained below the thermocline by a 
downward-refracting sound speed profile is scattered into adjacent higher modes (2-7) within the first half of the solitary wave, then abruptly couples to nonadjacent higher modes $(8-25)$ in the second half of the ISW and shelfbreak. This two-stage mode coupling process requires the ISW to be in close proximity to the sloping bathymetry; otherwise, it will not occur. Numerical simulations demonstrate the degree to which the combined effect of the ISW approaching the shelfbreak can elevate the depth-averaged acoustic energy in the upper water column downrange from the oceanographic and bathymetric features. Good agreement between numerical modeling and in situ experimental data support the predictions.

\section{ACKNOWLEDGMENTS}

The ASIAEX and NLIWI experiments were supported jointly by the National Science Council of Taiwan and the U.S. Office of Naval Research. Special thanks are offered to all of the ASIAEX and NLIWI experiment participants for their successful efforts, to the lead Principal Investigators from the U.S. and Taiwan, and to the crew members of the research vessels R/V OR1, R/V OR2, and R/V OR3. Special thanks are also offered to Dr. Ying-Tsong Lin for his useful comments on this manuscript. This work is supported by the National Science Council of Taiwan with project No. NSC 100-2611-M-110-017-.

Abawi, A. T. (2002). "An energy-conserving one-way coupled mode propagation model," J. Acoust. Soc. Am. 111(1), 160-167.

Abawi, A. T., Collins, M. D., and Kuperman, W. A. (1997). "The coupled mode parabolic equation,” J. Acoust. Soc. Am. 102, 233-238.

Chiu, C. S., Ramp, S. R., Miller, C. W., Lynch, J. F., Duda, T. F., and Tang, T. Y. (2004). "Acoustic intensity fluctuations induced by South China Sea internal tides and solitons," IEEE J. Oceanic Eng. 29, 1249-1263.

Collins, M. D. (1988). FEPE User's Guide, NORDA Technical Note No. 365 (Naval Research Laboratory, Stennis Space Center, MS).

Colosi, J. A., Beardsley, R. C., Lynch, J. F., Gawarkiewicz, G., Chiu, C. S., and Scotti, A. (2001). "Observations of nonlinear internal waves on the outer New England continental shelf during the summer Shelfbreak Primer study," J. Geophys. Res. 106(C5), 9587-9601.

Duda, T. F. (2004). "Acoustic mode coupling by nonlinear internal wave packets in a shelfbreak front area," IEEE J. Oceanic Eng. 29, 118-125.

Duda, T. F., Lin, Y. T., and Reeder, D. B. (2011). "Observationally constrained modeling of sound in curved ocean internal waves: Examination of deep ducting and surface ducting at short range," J. Acoust. Soc. Am. 130(3), 1173-1187.

Duda, T. F., Lynch, J. F., Newhall, A. E., Wu, L., and Chiu, C. S. (2004). "Fluctuation of 400-Hz sound intensity in the 2001 ASIAEX South China Sea experiment," IEEE J. Oceanic Eng. 29, 1264-1279.

Duda, T. F., and Preisig, J. C. (1999). "A modeling study of acoustic propagation through moving shallow-water solitary wave packets," IEEE J. Oceanic Eng. 24(1), 16-32.

Evans, R. B. (1983). "A coupled mode solution for acoustic propagation in a waveguide with stepwise depth variations of a penetrable bottom," J. Acoust. Soc. Am. 74, 188-195.
Fawcett, J. A. (1992). "A derivation of the differential equations of coupledmode propagation,” J. Acoust. Soc. Am. 92(1), 290-295.

Jensen, F. B., and Kuperman, W. A. (1980). "Sound propagation in a wedge-shaped ocean with a penetrable bottom," J. Acoust. Soc. Am. 67, 1564-1566.

Jensen, F. B., Kuperman, W. A., Porter, M. B., and Schmidt, H. (1994). Computational Ocean Acoustics, 1st ed. (AIP, New York), Chap. 5, pp. 320-323.

Jensen, F. B., and Tindle, C. T. (1987). "Numerical modeling results for mode propagation in a wedge," J. Acoust. Soc. Am. 82, 211-216.

Liu, A. K. (1988). "Analysis of nonlinear internal waves in the New York Bight,” J. Geophys. Res. 93(C10), 12317-12329.

Liu, A. K., Ramp, S. R., Zhao, Y., and Tang, T. Y. (2004). "A case study of internal solitary wave propagation during ASIAEX 2001," IEEE J. Oceanic Eng. 29, 1144-1156

Lynch, J. F., Ramp, S. R., Chiu, C. S., Tang, T. Y., Yang, Y. J., and Simmen, J. A. (2004). "Research highlights from the Asian Seas International Acoustics Experiment in the South China Sea," IEEE J. Oceanic Eng. 29, 1067-1074.

Mackenzie, K. V. (1981). "Nine-term equation for sound speed in the oceans," J. Acoust. Soc. Am. 70(3), 807-812.

McDonald, B. E. (1996). "Bathymetric and volumetric contributions to ocean acoustic mode coupling," J. Acoust. Soc. Am. 100, 219-224.

Milder, D. M. (1969). "Ray and wave invariants for SOFAR channel propagation," J. Acoust. Soc. Am. 46, 1259-1263.

Nagl, A., Uberall, H., Haug, A. J., and Zarur, G. L. (1978). "Adiabatic mode theory of underwater sound propagation in a rangedependent environment," J. Acoust. Soc. Am. 63, 739-749.

Newhall, A., Costello, L., Duda, T., Dunn, J., Gawarkiewicz, G., Irish, J., Kemp, J., McPhee, N., Liberatore, S., Lynch, J., Ostrom, W., Schroeder, T., Trask, R., and van der Heydt, K. (2001). "Preliminary acoustic and oceanographic observations from the ASIAEX 2001 South China Sea experiment," Report No. WHOI-2001-12 (Woods Hole Oceanographic Institution, MA), pp. 38-47.

Pierce, A. D. (1965). "Extension of the method of normal modes to sound propagation in an almost-stratified medium," J. Acoust. Soc. Am. 37(1), 19-27.

Preisig, J. C., and Duda, T. F. (1997). "Coupled acoustic mode propagation through continental-shelf internal solitary waves," IEEE J. Oceanic Eng. 22, 256-269.

Ramp, S. R., Tang, T. Y., Duda, T. F., Lynch, J. F., Liu, A. K., Chiu, C. S., Bahr, F. L., Kim, H. R., and Yang, Y. J. (2004). "Internal solitons in the northeastern South China Sea. Part I: Sources and deep water propagation,” IEEE J. Oceanic Eng. 29, 1157-1181.

Reeder, D. B., Chui, Linus Y. S., and Chen, C. F. (2010). "Experimental evidence of horizontal refraction by nonlinear internal waves of elevation in shallow water in the South China Sea: 3D versus Nx2D acoustic propagation modeling," J. Comput. Acoust. 18, 267-278.

Rutherford, S. R., and Hawker, K. E. (1981). "Consistent coupled mode theory of sound propagation for a class of nonseparable problems," J. Acoust. Soc. Am. 70, 554-564.

Shang, E. C., and Wang, Y. Y. (1993). "Acoustic travel time computation based on PE solution,” J. Comput. Acoust. 1, 91-100.

Shroyer, E. L., Moum, J. N., and Nash, J. D. (2011). "Nonlinear internal waves over New Jersey's continental shelf," J. Geophys. Res. 116, C03022.

Stotts, S. A. (2002). "Coupled-mode solutions in generalized ocean environments," J. Acoust. Soc. Am. 111(4), 1623-1643.

Yang, Y. J., Tang, T. Y., Chang, M. H., Liu, A. K., Hsu, M. K., and Ramp, S. R. (2004). "Solitons northeast of Tung-Sha Island during the ASIAEX pilot studies," IEEE J. Oceanic Eng. 29, 1182-1199. 